\title{
GENERALIZAÇÃO DE ESTRATÉGIAS DE DESCONTAMINAÇÃO DE RETICULADOS DE AUTÔMATOS CELULARES
}

\author{
Marcelo A. NogueirA ${ }^{2}$, Pedro P.B. De OliveirA ${ }^{1,2}$ \\ Universidade Presbiteriana Mackenzie \\ ${ }^{1}$ Faculdade de Computação e Informática \& ${ }^{2}$ Pós-Graduação em Engenharia Elétrica \\ Rua da Consolação 896, Consolação - 01302-907 São Paulo, SP \\ marcelo.arboriegmail.com, pedrobemackenzie.br
}

\begin{abstract}
Here we expand the limits imposed by Daadaa (2012) about the decontamination process of the lattice of a twodimensional cellular automaton, by understanding the characteristics of the rules proposed therein. It was possible to identify two general rules for each type of neighborhood addressed, and to show that they outperform those by Daadda (2012), in terms of their efficacy on a set of randomly distributed initial configurations.
\end{abstract}

Keywords - Cellular automaton, network decontamination, discrete dynamical system, two-dimensional neighbourhoods.

\begin{abstract}
Resumo - Expandem-se aqui os limites impostos por Daadaa (2012) a respeito do processo de descontaminação do reticulado de um autômato celular bidimensional, a partir de um maior entendimento das características das regras lá propostas. Foi possível identificar duas regras gerais para cada tipo de vizinhança utilizado, e mostrar que, para condições iniciais aleatórias, tais regras possuem eficácia superior que as definidas por Daadaa (2012).
\end{abstract}

Palavras-chave - Autômato celular, descontaminação de rede, sistema dinâmico discreto, vizinhança bidimensional.

\section{Introdução}

Um autômato celular é um conjunto de células em uma grade com forma especificada que se desenvolve através de uma série de passos discretos de tempo de acordo com um conjunto de regras com base nos estados das células vizinhas. As regras são aplicadas de forma iterativa por quantas vezes desejarmos (Wolfram, 2002). O autômato celular considerado neste artigo é um autômato celular bidimensional de três estados, onde a transição para o estado descontaminado prove à célula um tempo de imunidade no qual seu estado permanece descontaminado. O problema de descontaminar a grade consiste em aplicar uma regra que em tempo finito leve toda a grade para o mesmo estado.

Descrevem-se aqui resultados obtidos ao medir a eficácia de regras dos autômatos celulares definidos por Daadaa (2012), regras estas capazes de descontaminar reticulados bidimensionais com condições iniciais pré-definidas. Com premissas rígidas para as regras e condições iniciais (CI) foi possível provar teoremas de que as regras são ótimas para descontaminar as CIs previstas. Mas não se sabe quais outras CIs são passíveis de descontaminação.

A principal premissa flexibilizada para medir a eficácia das regras foi a exigência de que na CI todo o reticulado esteja contaminado. As regras ótimas foram aplicadas em classes de CIs, uma classe para cada regra ótima. A eficácia foi medida pelo número de CIs destas classes que foram descontaminadas pela regra ótima.

A partir da observação das transições aplicadas às condições de estados pelas regras ótimas, se podem observar quais transições de estado é interessan- te para descontaminar um reticulado, a depender do tipo de vizinhança adotado. Com o conjunto destas transições de estado foi possível construir duas regras. Uma ligada a vizinhança de von Neumann e outra à vizinhança de Moore.

Cada regra construída foi aplicada às mesmas classes de CIs nas quais as regras ótimas foram aplicadas e se pode observar que em geral a eficácia foi maior, apesar de alguns casos não apresentar variação ou pouca variação na eficácia.

Por fim as regras construídas foram aplicadas a CIs com dimensões e distribuições de estados aleatórios. Percebeu-se uma eficácia virtualmente total das regras nesta situação. No entanto não se sabe qual o comportamento da eficácia se as regras construídas fossem aplicadas em CIs com distribuição uniforme de estados.

\section{O Problema de Descontaminação de Rede Bidimensional e a Eficácia das Regras Ótimas}

O problema de descontaminação de redes bidimensionais pode ser caracterizado por uma rede totalmente contaminada e procura-se uma estratégia para que, após um tempo finito, todas as células da rede estejam com seu estado normal, descontaminado. A Erro! Fonte de referência não encontrada. mostra a nomenclatura e codificação utilizada para os estados neste artigo.

Tabela 1: Nomenclatura e codificação para os estados das células.

\begin{tabular}{|l|r|l|}
\hline Estado & Valor & Cor \\
\hline Descontaminado & 0 & Cinza \\
\hline Contaminado & 1 & Branco \\
\hline Descontaminando & 2 & Preto \\
\hline
\end{tabular}


Este trabalho aborda a descontaminação básica e temporal. No caso da descontaminação temporal uma célula passa por um tempo de imunidade, no qual ela não pode ser contaminada. Além disso, o ambiente pode possuir uma condição de contorno finita ou periódica. Cada uma das regras com seu tempo de imunidade associado foram provados ótimos para descontaminar as suas respectivas CIs, neste trabalho estas CIs são as CIs de referência. Para provar ótimos a regra e o tempo de imunidade foram assumidas premissas rígidas, permitindo provar os teoremas enunciados (Daadaa, 2012).

\subsection{Descontaminação de Rede por Autômatos Celu- lares}

Autômatos celulares são modelos que se prestam a descrever sistemas e padrões complexos através de componentes simples que através do seu comportamento local aos componentes, emerge o comportamento complexo descrito pelo modelo (Packard e Wolfram, 1985).

O ambiente, alvo da descontaminação, é um ambiente discreto formado por células interligadas de forma regular formando um reticulado bidimensional retangular. Cada célula deste reticulado está em um estado que será alterado mediante uma regra, selecionada para a tarefa de descontaminação, a depender do estado de suas células vizinhas. A Tabela 2 mostra dois exemplos de transições de estado que podem fazer parte de uma regra. A primeira linha apresenta uma transição de estado para uma vizinhança de Moore e a segunda para uma vizinhança de von Neumann. O primeiro estado da vizinhança é o estado da célula central, o segundo da célula à esquerda seguindo-se no sentido horário até a última célula da vizinhança.

Tabela 2: Exemplo de transições de estado para uma vizinhança de Moore na primeira linha e para uma vizinhança de von Neuman na segunda linha.

\begin{tabular}{|c|c|}
\hline Vizinhança & Novo Estado \\
\hline$\{1,2,0,0,1,0,1,0,0\}$ & 0 \\
\hline$\{1,2,0,1,1\}$ & 2 \\
\hline
\end{tabular}

Se for encarado $a_{i}^{t}$ como o valor de uma célula $i$ no instante $t$, pode-se encarar a mudança do valor de uma célula como uma transição do estado da célula no instante $t$ para o novo valor em $t+1$ como o resultado da aplicação de uma função de transição que recebe um vetor com os estados da vizinhança e devolve o novo valor da célula central, fazendo $a_{i}^{t+1}=f\left(\overrightarrow{a^{t}}\right)$. A Figura 1 mostra a transição de estado de uma célula central em um autômato de três estados, onde a transição para a nova configuração em $a_{i}^{t+1}$ é feita devido aos estados das células em $a_{i}^{t}$ (Packard e Wolfram, 1985).

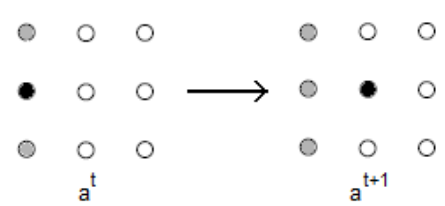

Figura 1: Exemplo de transição de estado de uma célula central em um autômato celular de 3 estados.

Há dois tipos de vizinhança abordadas neste artigo para se fazer a transição de estado, uma vizinhança de von Neumann ou de Moore, conforme mostra a Figura 2.
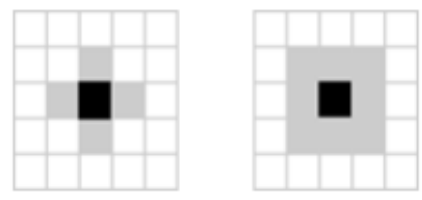

Figura 2: Tipos de vizinhança. À esquerda vizinhança de von Neumann e à direita vizinhança de Moore.

Uma célula que foi descontaminada pode voltar ao estado contaminado caso haja pelo menos uma célula contaminada em sua vizinhança. Além do tipo da vizinhança é importante levar em consideração o tempo de imunidade que é o tempo que uma célula descontaminada permanece neste estado mesmo em contato com células contaminadas em sua vizinhança.

Devido ao tempo de imunidade há dois processos de descontaminação a considerar: descontaminação básica; sem tempo de imunidade. E a descontaminação temporal; com tempo de imunidade. As próximas seções tratam destes dois casos sob a ótica das regras ótimas definidas por Daadaa (2012).

\subsection{Descontaminação Básica e Eficácia das Regras}

Daadaa (2012) definiu suas regras e provou que elas são ótimas analiticamente através de teoremas para cada uma delas. Para verificar a eficácia de tais regras, cada uma delas foi aplicada em uma classe de CIs derivada da CIs de referência. Os teoremas são enunciados a seguir.

\section{Teorema 4.1:}

Descontaminação básica ótima pode ser obtida em um autômato celular finito de duas dimensões e vizinhança de von Neumann, usando $n$ células simultâneas no estado descontaminando (Daadaa, 2012).

Tabela 3: Regra para a descontaminação básica em um autômato celular finito com vizinhança de von Neumann (Daadaa, 2012).

\begin{tabular}{|c|c|}
\hline Vizinhança & Novo Estado \\
\hline$\left\{2,{ }^{*},{ }^{*}, *\right\}$ & 0 \\
\hline$\{1,2,0,1,1\}$ & 2 \\
\hline$\{1,2,0,0,1\}$ & 2 \\
\hline$\{1,2,1,1,1\}$ & 2 \\
\hline$\{1,2,1,1,0\}$ & 2 \\
\hline$\{1,2,1,0,1\}$ & 2 \\
\hline$\{1,2,1,0,0\}$ & 2 \\
\hline
\end{tabular}




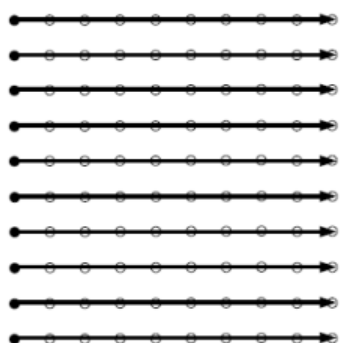

Figura 3: Descontaminação básica em um autômato celular bidimensional finito com vizinhança de von Neumann (Daadaa, 2012)

\section{Teorema 4.2:}

A descontaminação básica ótima pode ser obtida em um autômato celular circular, com a vizinhança de von Neumann, usando $2 n$ células simultâneas descontaminando (Daadaa, 2012).

Tabela 4:Regra para a descontaminação básica em um autômato celular circular de duas dimensões com vizinhança de von Neumann (Daadaa, 2012).

\begin{tabular}{|c|c|}
\hline Vizinhança & Novo Estado \\
\hline$\left\{2,{ }^{*},{ }^{*},{ }^{*}\right\}$ & 0 \\
\hline$\{1,2,1,1,1\}$ & 2 \\
\hline$\{1,1,1,2,1\}$ & 2 \\
\hline$\{1,2,1,2,1\}$ & 2 \\
\hline
\end{tabular}

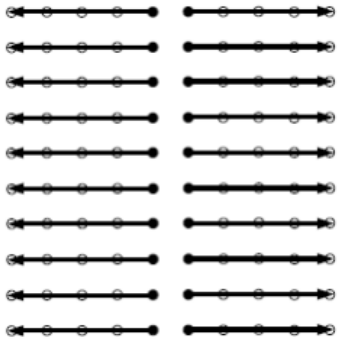

Figura 4:Descontaminação básica em um autômato celular bidimensional circular com vizinhança de von Neumann (Daadaa, 2012)

Para gerar as classes de condições iniciais para cada regra foram mantidos os estados das células descontaminando e se variou para todas as possibilidades entre o estado contaminado e o descontaminado nas demais células. Nestes dois casos as regras não se mostraram muito eficazes.

Tabela 5: Eficácia das regras da descontaminação básica

\begin{tabular}{|c|c|c|c|}
\hline Teorema & Cls Possiveis & Cls Encontradas & Eficácia \\
\hline 4.1 & 1048576 & 1036 & $0,10 \%$ \\
\hline 4.2 & 16777216 & 1 & $0,00 \%$ \\
\hline
\end{tabular}

\subsection{Descontaminação Temporal e Eficácia das Re- gras}

Daadaa (2012) também definiu regras para uma descontaminação temporal, provadas ótimas segundo os teoremas a seguir.

\section{Teorema 4.3}

Com uma simples célula no estado descontaminando por unidade de tempo, a descontaminação temporal é possivel se, e somente se, o tempo de imunidade for $t \geq 4(n-1)-1$ (Daadaa, 2012).
Tabela 6: Regra para a descontaminação temporal com uma única célula descontaminando em um autômato celular finito com

\begin{tabular}{|c|c|}
$\begin{array}{c}\text { Vizinhinhança } \\
\text { Novo Estado }\end{array}$ \\
\hline$\left\{2,{ }^{*},{ }^{*}\right.$, \\
\hline$\{1,2,1,1,0\}$ & 0 \\
\hline$\{1,2,1,0,0\}$ & 2 \\
\hline$\{1,0,2,1,1\}$ & 2 \\
\hline$\{1,0,2,1,0\}$ & 2 \\
\hline$\{1,0,2,0,0\}$ & 2 \\
\hline$\{1,0,0,2,1\}$ & 2 \\
\hline$\{1,1,0,2,1\}$ & 2 \\
\hline$\{1,1,1,0,2\}$ & 2 \\
\hline$\{1,1,0,0,2\}$ & 2 \\
\hline$\{1,0,0,0,2\}$ & 2 \\
\hline
\end{tabular}

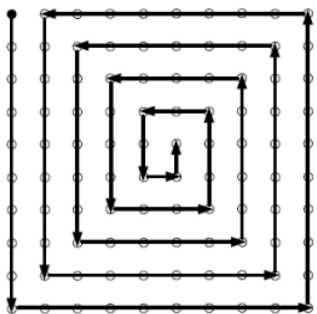

Figura 5: Propagação de uma simples célula descontaminando com tempo de imunidade e vizinhança de von Neumann (Daadaa, 2012).

\section{Teorema 4.4}

Com $k=2$ ou $k=4$ células descontaminando por unidade de tempo, a descontaminação temporal é possivel se, e somente se, o tempo de imunidade for $t \geq \frac{4}{k}(n-1)-1$ (Daadaa, 2012).

Tabela 7: Regra para a descontaminação temporal com duas células descontaminando em um autômato celular finito com

\begin{tabular}{|c|c|}
\multicolumn{2}{|c|}{ Vizinhança de von Neumann (Daadaa, 2012} \\
\hline$\left\{2,{ }^{*},{ }^{*},{ }^{*}\right\}$ & Novo Estado \\
\hline$\{1,2,1,1,0\}$ & 0 \\
\hline$\{1,2,1,0,0\}$ & 2 \\
\hline$\{1,0,2,1,1\}$ & 2 \\
\hline$\{1,0,2,1,0\}$ & 2 \\
\hline$\{1,1,0,2,1\}$ & 2 \\
\hline$\{1,0,0,2,1\}$ & 2 \\
\hline$\{1,1,1,0,2\}$ & 2 \\
\hline$\{1,1,0,0,2\}$ & 2 \\
\hline$\{1,2,0,0,2\}$ & 2 \\
\hline$\{1,0,2,2,0\}$ & 2 \\
\hline$\{1,0,2,0,2\}$ & 2 \\
\hline
\end{tabular}




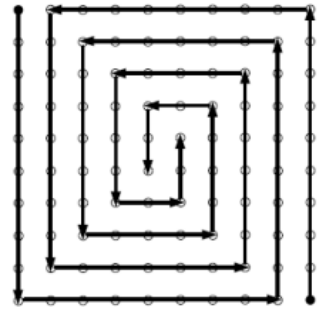

Figura 6:Propagação de duas células descontaminando com tempo de imunidade e vizinhança de von Neumann (Daadaa, 2012).

Tabela 8:Regra para a descontaminação temporal com quatro células descontaminando em um autômato celular finito com vizinhança de von Neumann (Daadaa, 2012)

\begin{tabular}{|c|c|}
\hline Vizinhança & Novo Estado \\
\hline$\{2, *, *, *\}$ & 0 \\
\hline$\{1,2,1,1,0\}$ & 2 \\
\hline$\{1,2,1,0,0\}$ & 2 \\
\hline$\{1,0,2,1,1\}$ & 2 \\
\hline$\{1,0,2,1,0\}$ & 2 \\
\hline$\{1,1,0,2,1\}$ & 2 \\
\hline$\{1,0,0,2,1\}$ & 2 \\
\hline$\{1,1,1,0,2\}$ & 2 \\
\hline$\{1,1,0,0,2\}$ & 2 \\
\hline$\{1,2,1,2,0\}$ & 2 \\
\hline$\{1,2,0,2,1\}$ & 2 \\
\hline$\{1,0,2,1,2\}$ & 2 \\
\hline$\{1,1,2,0,2\}$ & 2 \\
\hline$\{1,2,2,2,2\}$ & 2 \\
\hline
\end{tabular}

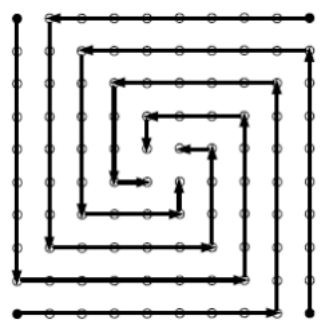

Figura 7: Propagação de quatro células descontaminando com tempo de imunidade e vizinhança de von Neumann (Daadaa, 2012).

\section{Teorema 4.5}

Seja $d_{\max }$ a distância máxima entre duas células descontaminando consecutivas ou entre uma célula descontaminando e um canto (do reticulado) no tempo 0. Se o número inicial de células descontaminando for ao menos 2, a regra da Tabela 9 obtém a descontaminação com tempo de imunidade $t=d_{\max }$ (Daadaa, 2012).

Tabela 9: Regra para descontaminação temporal com múltiplas células descontaminando em um autômato celular finito com vizinhança de Moore. (Daadaa, 2012).

\begin{tabular}{|c|c|}
\hline Vizinhança & Novo Estado \\
\hline$\left\{2 ; *^{*} ; * ; * *^{*} ; * ; *\right\}$ & 0 \\
\hline$\{1 ; 2 ; 0 ; 1 ; 1 ; 1 ; 1 ; 1 ; 0\}$ & 2 \\
\hline$\{1 ; 2 ; 0 ; 0 ; 0 ; 1 ; 1 ; 0 ; 0\}$ & 2 \\
\hline$\{1 ; 2 ; 0 ; 1 ; 1 ; 1 ; 0 ; 0 ; 0\}$ & 2 \\
\hline$\{1 ; 2 ; 0 ; 0 ; 0 ; 1 ; 1 ; 1 ; 0\}$ & 2 \\
\hline$\{1 ; 2 ; 0 ; 1 ; 1 ; 1 ; 1 ; 1 ; 2\}$ & 2 \\
\hline$\{1 ; 2 ; 0 ; 1 ; 0 ; 0 ; 0 ; 1 ; 0\}$ & 2 \\
\hline$\{1 ; 2 ; 0 ; 1 ; 0 ; 0 ; 0 ; 0 ; 0\}$ & 2 \\
\hline
\end{tabular}

\begin{tabular}{|l|l|}
\hline$\{1 ; 2 ; 0 ; 0 ; 0 ; 0 ; 0 ; 1 ; 0\}$ & 2 \\
\hline$\{1 ; 2 ; 2 ; 1 ; 1 ; 1 ; 1 ; 1 ; 0\}$ & 2 \\
\hline$\{1 ; 2 ; 0 ; 1 ; 0 ; 0 ; 0 ; 1 ; 2\}$ & 2 \\
\hline$\{1 ; 2 ; 2 ; 1 ; 0 ; 0 ; 0 ; 1 ; 0\}$ & 2 \\
\hline$\{1 ; 2 ; 2 ; 0 ; 0 ; 0 ; 0 ; 0 ; 0\}$ & 2 \\
\hline$\{1 ; 0 ; 0 ; 2 ; 0 ; 0 ; 0 ; 0 ; 0\}$ & 2 \\
\hline$\{1 ; 0 ; 0 ; 2 ; 1 ; 1 ; 1 ; 1 ; 0\}$ & 2 \\
\hline$\{1 ; 0 ; 0 ; 2 ; 1 ; 1 ; 0 ; 0 ; 0\}$ & 2 \\
\hline$\{1 ; 0 ; 0 ; 2 ; 0 ; 0 ; 0 ; 1 ; 0\}$ & 2 \\
\hline$\{1 ; 0 ; 0 ; 2 ; 1 ; 1 ; 1 ; 2 ; 0\}$ & 2 \\
\hline$\{1 ; 0 ; 0 ; 2 ; 0 ; 0 ; 0 ; 2 ; 0\}$ & 2 \\
\hline$\{1 ; 0 ; 0 ; 1 ; 0 ; 0 ; 0 ; 2 ; 0\}$ & 2 \\
\hline$\{1 ; 0 ; 0 ; 0 ; 0 ; 0 ; 0 ; 2 ; 0\}$ & 2 \\
\hline$\{1 ; 0 ; 0 ; 0 ; 0 ; 1 ; 1 ; 2 ; 0\}$ & 2 \\
\hline$\{1 ; 0 ; 0 ; 1 ; 1 ; 1 ; 1 ; 2 ; 0\}$ & 2 \\
\hline
\end{tabular}

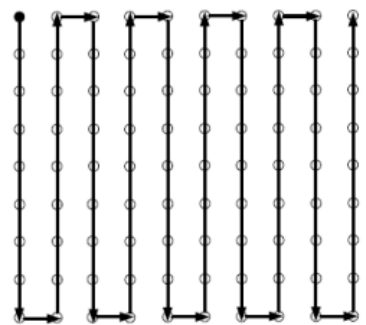

Figura 8:Propagação de uma única célula descontaminando, com tempo de imunidade e vizinhança de Moore (Daadaa, 2012).

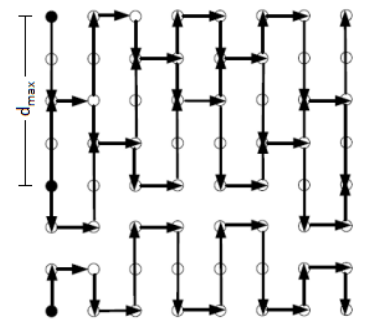

Figura 9:Propagação de múltiplas células descontaminando com tempo de imunidade e vizinhança de Moore (Daadaa, 2012).

\section{Teorema 4.9}

Com 1, 2, ou 4 células descontaminando por unidade de tempo, a descontaminação temporal em um autômato celular circular com vizinhança de von Neumann é impossível de ser obtida, independente do tempo de imunidade (Daadaa, 2012).

E deste teorema conclui-se o Corolário 4.10.

\section{Corolário 4.10}

Com $k<n$ células descontaminando por unidade de tempo, a descontaminação temporal em um autômato celular circular com vizinhança de von Neumann não é possivel, independente do tempo de imunidade (Daadaa, 2012).

\section{Teorema 4.11}

Seja $d_{\max }$ a distância máxima entre duas células descontaminando consecutivas, ou entre a célula descontaminando e um canto no tempo 0. Se o número inicial de células descontaminando é pelo menos 2, a regra da Tabela 10: Regra para descontaminação temporal com múltiplas células descontaminando em um autômato celular 
circular com vizinhança de Moore (Daadaa, 2012).Tabela 10 obtém a descontaminação em um autômato celular circular com tempo de imunidade $t=d_{\max }$ (Daadaa, 2012).

Tabela 10: Regra para descontaminação temporal com múltiplas células descontaminando em um autômato celular circular com

\begin{tabular}{|c|c|} 
vizinhança de Moore (Daadaa, 2012). \\
\hline Vizinhança & Novo Estado \\
\hline$\{2 ; * ; * ; * ; ; * ; * ;\}$ & 0 \\
\hline$\{1 ; 1 ; 1 ; 2 ; 1 ; 1 ; 1 ; 1 ; 1\}$ & 2 \\
\hline$\{1 ; 1 ; 1 ; 1 ; 1 ; 1 ; 1 ; 2 ; 1\}$ & 2 \\
\hline$\{1 ; 1 ; 1 ; 2 ; 1 ; 1 ; 1 ; 2 ; 1\}$ & 2 \\
\hline$\{1 ; 2 ; 0 ; 1 ; 1 ; 1 ; 1 ; 1 ; 2\}$ & 2 \\
\hline$\{1 ; 2 ; 0 ; 1 ; 1 ; 1 ; 1 ; 1 ; 0\}$ & 2 \\
\hline$\{1 ; 1 ; 1 ; 1 ; 0 ; 2 ; 0 ; 1 ; 1\}$ & 2 \\
\hline$\{1 ; 1 ; 1 ; 1 ; 2 ; 2 ; 0 ; 1 ; 1\}$ & 2 \\
\hline$\{1 ; 0 ; 0 ; 2 ; 1 ; 1 ; 1 ; 1 ; 0\}$ & 2 \\
\hline$\{1 ; 0 ; 0 ; 1 ; 1 ; 1 ; 1 ; 2 ; 0\}$ & 2 \\
\hline$\{1 ; 0 ; 0 ; 2 ; 1 ; 1 ; 1 ; 2 ; 0\}$ & 2 \\
\hline$\{1 ; 1 ; 1 ; 1 ; 0 ; 0 ; 0 ; 2 ; 1\}$ & 2 \\
\hline$\{1 ; 1 ; 1 ; 2 ; 0 ; 0 ; 0 ; 1 ; 1\}$ & 2 \\
\hline$\{1 ; 1 ; 1 ; 2 ; 0 ; 0 ; 0 ; 2 ; 1\}$ & 2 \\
\hline$\{1 ; 2 ; 0 ; 1 ; 0 ; 0 ; 0 ; 1 ; 0\}$ & 2 \\
\hline$\{1 ; 0 ; 0 ; 1 ; 2 ; 2 ; 0 ; 1 ; 2\}$ & 2 \\
\hline$\{1 ; 0 ; 0 ; 1 ; 0 ; 2 ; 0 ; 1 ; 2\}$ & 2 \\
\hline$\{1 ; 0 ; 0 ; 2 ; 1 ; 2 ; 0 ; 1 ; 0\}$ & 2 \\
\hline$\{1 ; 0 ; 0 ; 1 ; 0 ; 2 ; 1 ; 2 ; 0\}$ & 2 \\
\hline$\{1 ; 2 ; 1 ; 2 ; 0 ; 0 ; 0 ; 1 ; 0\}$ & 2 \\
\hline$\{1 ; 0 ; 0 ; 1 ; 2 ; 2 ; 0 ; 1 ; 2\}$ & 2 \\
\hline$\{1 ; 2 ; 0 ; 1 ; 0 ; 0 ; 0 ; 1 ; 1\}$ & 2 \\
\hline$\{1 ; 0 ; 0 ; 1 ; 2 ; 1 ; 1 ; 2 ; 0\}$ & 2 \\
\hline$\{1 ; 1 ; 2 ; 1 ; 0 ; 0 ; 0 ; 2 ; 1\}$ & 2 \\
\hline$\{1 ; 2 ; 0 ; 1 ; 0 ; 2 ; 0 ; 1 ; 0\}$ & 2 \\
\hline$\{1 ; 0 ; 0 ; 2 ; 0 ; 0 ; 0 ; 1 ; 0\}$ & 2 \\
\hline$\{1 ; 0 ; 0 ; 1 ; 0 ; 0 ; 0 ; 2 ; 0\}$ & 2 \\
\hline$\{1 ; 0 ; 0 ; 2 ; 0 ; 0 ; 0 ; 2 ; 0\}$ & 2 \\
\hline$\{1 ; 0 ; 0 ; 0 ; 0 ; 0 ; 0 ; 2 ; 0\}$ & 2 \\
\hline$\{1 ; 0 ; 0 ; 2 ; 0 ; 0 ; 0 ; 0 ; 0\}$ & 2 \\
\hline
\end{tabular}

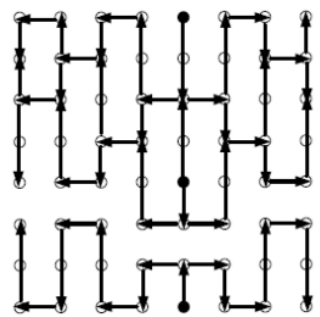

Figura 10: Propagação de múltiplas células descontaminando com tempo de imunidade em um autômato celular periódico e vizinhança de Moore (Daadaa, 2012).

Tabela 11: Eficácia das regras da descontaminação temporal.

\begin{tabular}{|l|r|r|r|}
\hline Teorema & Cls Possíveis & Cls Encontradas & Eficácia \\
\hline 4.3 & 16777216 & 2755411 & $16,42 \%$ \\
\hline $4.4-2$ & 8388608 & 360 & $0,00 \%$ \\
\hline $4.4-4$ & 2097152 & 3676 & $0,18 \%$ \\
\hline $4.5-1$ & 16777216 & 665981 & $3,97 \%$ \\
\hline $4.5-3$ & 536870912 & 8 & $0,00 \%$ \\
\hline 4.11 & 8589934592 & 0 & $0,00 \%$ \\
\hline
\end{tabular}

Aqui é importante ressaltar que não foi observado o proposto para o teorema 4.11 e por isso a quantidade de CIs encontradas permaneceu em 0 . A Figura
11 mostra a dinâmica obtida em t2 que contraria a dinâmica proposta na Figura 10.

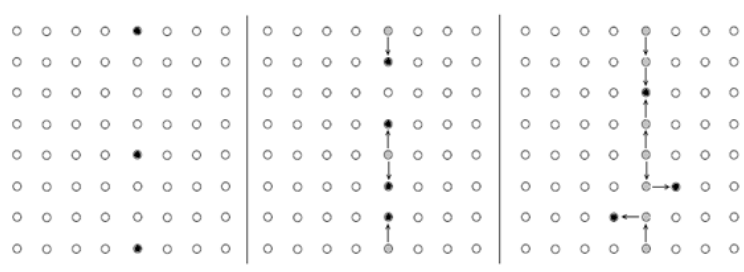

Figura 11: Dinâmica de descontaminação observada em $\mathrm{t}_{2}$ com as regras da Tabela 10

\section{Construção de Regras Mais Eficazes}

Observando as transições de estado das várias regras definidas por Daadaa (2012) é possível construir regra mais eficazes. Foram definidos 5 princípios que tornam uma transição adequada ao processo de descontaminação.

(1) Toda célula no estado 2 em $t$ deve passar para o estado $0 \mathrm{em} \mathrm{t}+1$. (2) Toda célula que transita de 2 para 0 deve possuir pelo menos uma célula no estado 2 em sua vizinhança. (3) Uma célula que transita de 0 para 1 , necessariamente possui uma célula no estado 1 em sua vizinhança e seu tempo de imunidade é 0. (4) Para toda transição do estado 1 para o 2 deverá haver um estado 2 na vizinhança em t-1. (5) Caso alguma célula que transita de 2 para 0 não possua uma célula no estado 2 em sua vizinhança em $\mathrm{t}+1$, esta deve ser a última transição e o ambiente deve estar descontaminado.

Para descontaminar a rede as transições de estado que levam a célula ao estado 1 não são desejadas. Todas as transições para 0 são encaradas como uma única transição como por exemplo a primeira linha da Tabela 10.

Na vizinhança de Moore há $3^{9}$ células e na de von Neumann há $3^{5}$. Como as transições para 0 foram tratadas como uma só, e que as transições para 1 não interessam, para uma vizinhança de Moore há $3^{9}$ + 1 transições de estado passíveis de comporem regras para a descontaminação e $3^{5}+1$ para uma vizinhança de von Neumann. Aplicando os 5 princípios que foram estabelecidos, esta quantidade cai para 6178 transições de estados para uma vizinhança de Moore e 66 para uma vizinhança de von Neumann.

As 6178 transições de estado para vizinhança de Moore e 66 para a vizinhança de von Neumann formam um conjunto de transições para cada tipo de vizinhança e subconjuntos destas transições formam regras que podem descontaminar uma rede. No entanto o próprio conjunto de cada vizinhança forma uma regra. Os resultados da analise da eficácia destas regras é abordado na próxima seção.

\subsection{Descontaminação Pelas Regras Construídas}

As mesmas experiências descritas nas seções 2.2 e 2.3 foram repetidas com as mesmas classes de 
CIs com as regras construídas para as vizinhanças de Moore e von Neumann. As tabelas 12 e 13 mostram os resultados.

Tabela 12: Eficácia para a regra para a vizinhança de von Neumann.

\begin{tabular}{|l|r|r|r|}
\hline Teorema & Cls Possíveis & Cls Encontradas & Eficácia \\
\hline 4.1 & 1048576 & 1048 & $0,10 \%$ \\
\hline 4.2 & 16777216 & 4 & $0,00 \%$ \\
\hline 4.3 & 16777216 & 12582898 & $75,00 \%$ \\
\hline $4.4-2$ & 8388608 & 2880987 & $34,34 \%$ \\
\hline $4.4-4$ & 2097152 & 90321 & $4,31 \%$ \\
\hline $4.5-1$ & 16777216 & 665981 & $3,97 \%$ \\
\hline $4.5-3$ & 536870912 & 339051324 & $63,15 \%$ \\
\hline 4.11 & 8589934592 & 0 & $0,00 \%$ \\
\hline
\end{tabular}

Tabela 13: Eficácia para a regra para a vizinhança de Moore

\begin{tabular}{|l|r|r|r|}
\hline Teorema & Cls Possiveis & Cls Encontradas & Eficácia \\
\hline 4.1 & 1048576 & 279072 & $26,61 \%$ \\
\hline 4.2 & 16777216 & 452 & $0,00 \%$ \\
\hline 4.3 & 16777216 & 12582602 & $75,00 \%$ \\
\hline $4.4-2$ & 8388608 & 6291713 & $75,00 \%$ \\
\hline $4.4-4$ & 2097152 & 1042056 & $49,69 \%$ \\
\hline $4.5-1$ & 16777216 & 12582585 & $75,00 \%$ \\
\hline $4.5-3$ & 536870912 & 269611795 & $50,22 \%$ \\
\hline 4.11 & 8589934592 & 35960416 & $26,79 \%$ \\
\hline
\end{tabular}

É possível observar que em geral as regras construídas possuem eficácia superior às regras ótimas definidas por Daadaa (2012). Mas este valor ocorre para as classes de condição iniciais derivadas das condições iniciais de referência. A próxima seção mostra a eficácia das regras construídas para condições iniciais aleatórias.

\subsection{Descontaminação de condições Iniciais Aleató- rias Pelas Regras Construídas}

Com o objetivo de descobrir qual a eficácia das regras construídas para condições iniciais aleatórias foram geradas grupos de 300.000 CIs aleatórias, tanto na distribuição dos estados quanto nas dimensões do reticulado, para cada tipo de vizinhança e condição de contorno do AC. E observou-se que a eficácia é praticamente total, pois são todas superiores a $99 \%$. A Tabela 14 resume os resultados.

Tabela 14: Eficácias para descontaminação de condições iniciais aleatórias pelas regras aqui construídas.

\begin{tabular}{|c|c|c|c|c|}
\hline Vizinhança & $\begin{array}{l}\text { Condição de } \\
\text { Contorno }\end{array}$ & Cls & $\begin{array}{l}\text { Descon- } \\
\text { tamina- }\end{array}$ & Eficácia \\
\hline \multirow{2}{*}{ Moore } & Finita & 300.000 & 299.425 & $99,81 \%$ \\
\hline & Periódica & 300.000 & 299.504 & $99,83 \%$ \\
\hline \multirow{2}{*}{ von Neumann } & Finita & 300.000 & 299.432 & $99,81 \%$ \\
\hline & Periódica & 300.000 & 299.528 & $99,84 \%$ \\
\hline
\end{tabular}

É importante observar que ao gerar condições iniciais aleatórias para verificar a eficácia das regras aqui construídas, foram geradas condições iniciais com distribuição uniforme para os 3 estados. Por isso a quantidade máxima de entidades nas condições iniciais gira em torno de $33 \%$ das células do reticulado.

\section{Conclusão}

Daadaa (2012) definiu regras ótimas para descontaminar as CIs propostas, mas pelos resultados de nossa investigação fica evidente que há outras CIs que podem ser descontaminadas por estas mesmas regras.

O fato de ter sido possível mostrar experimentalmente o resultado positivo das regras gerais aqui propostas em condições iniciais com distribuição Normal de seus estados, resta verificar esse efeito com distribuição uniforme. Afinal, como a literatura mostra, um fato não implica no outro, como se vê na tarefa de classificação de densidade de ACs binários (em condição de contorno periódica), em que o objetivo é determinar se há quantidade maior de $1 \mathrm{~s}$ ou de 0s numa CI arbitrária; observa-se nesse caso que CIs com distribuição uniforme de estados são mais facilmente classificadas do que quando a distribuição é Normal (Wolz e de Oliveira, 2008).

Apesar de a verificação da eficácia das regras gerais aqui propostas para uma distribuição uniforme ainda carecer de verificação, o fato de eficácia para as regras gerais ser menor para as CIs de referência é uma indicação de que na distribuição uniforme a eficácia será menor quando o percentual de células descontaminando for menor que $33 \%$ e provavelmente crescerá quando o percentual se aproximar de uma distribuição normal.

\section{Referências Bibliográficas}

Daadaa, Yassine (2012). Network decontamination with temporal immunity. Thesis ( $\mathrm{PhD}$ in Computer Science) - School of Electrical Engineering and Computer Science, University of Ottawa. Ottawa, Canadá.

Packard, Norman H.; Wolfram, Stephen (1985). Two-dimensional cellular automata. Journal of Statistical Physics. 38(5-6). Princeton, Estados Unidos, pp. 901-946.

Wolfram, S. (2002). A new kind of science. Wolfram Media, pp. 23 - 50.

Wolz, Dietmar; de Oliveira, Pedro P.B. (2008). Very Effective evolutionary techniques for searching cellular automata rule spaces. Journal of Cellular Automata, 3(4):289-312. 